\title{
Identifying Graph Automorphisms Using Determining Sets
}

\author{
Debra L. Boutin \\ Department of Mathematics \\ Hamilton College, Clinton, NY 13323 \\ dboutin@hamilton.edu
}

Submitted: May 31, 2006; Accepted: Aug 22, 2006; Published: Sep 7, 2006

Mathematics Subject Classification: 05C25

\begin{abstract}
A set of vertices $S$ is a determining set for a graph $G$ if every automorphism of $G$ is uniquely determined by its action on $S$. The determining number of a graph is the size of a smallest determining set. This paper describes ways of finding and verifying determining sets, gives natural lower bounds on the determining number, and shows how to use orbits to investigate determining sets. Further, determining sets of Kneser graphs are extensively studied, sharp bounds for their determining numbers are provided, and all Kneser graphs with determining number 2, 3, or 4 are given.
\end{abstract}

\section{Introduction}

You are a contestant on the newest mathematical game show "What's my symmetry?" The host gives you a graph and an anonymous automorphism $\sigma$. You are allowed to choose vertices and ask for their images under $\sigma$. Your goal is to determine the automorphism by correctly identifying the images of the remaining vertices. The amount of money you can win is inversely proportional to the number of vertices whose images you ask to see. In the bonus round, you are asked to correctly identify the automorphism group of the graph.

The set of vertices you wish to choose is called a determining set and the smallest size of such a set is called the determining number of the graph. As you study in preparation for the game, some questions you ask are: How can we find a determining set? Given a set of vertices, how can we decide whether it is determining? Can we use $\operatorname{Aut}(G)$ to find a determining set for $G$ ? Can we use a determining set to find $\operatorname{Aut}(G)$ ? How small can a determining set be? How large might it be? What properties of the vertex orbits make it easy to find a determining set or to bound the determining number? These questions are addressed in this paper.

The paper is organized as follows. Formal definitions of determining sets and determining numbers, initial observations, and examples are given at the beginning of Section 2. Section 2.1 introduces some natural bounds on the determining number in terms of the size of the automorphism group. Methods of finding, or verifying, a determining set 
are discussed in Section 2.2, while Section 2.3 shows how to use orbits to find a determining set or to bound a determining number. Determining sets for the Kneser graphs are studied extensively in Section 3. In particular, sharp upper and lower bounds on their determining numbers are found in Section 3.1. Further, formulas are developed that aid in investigating determining sets for Kneser graphs, and all Kneser graphs with determining number 2, 3, and 4 are listed in Section 3.2. Open questions are given in Section 4.

It has come to my attention that independently Erwin and Harary [3] defined an equivalent set that they called the fixing set. Also, Collins and Laison [2], and Gibbons and Laison [4], are reporting results on these sets.

\section{Basics, Bounds, and Tools}

We have already introduced the idea of determining sets. The following is a more formal beginning to their study.

Definition 1. A subset $S$ of the vertices of a graph $G$ is called a determining set if whenever $g, h \in \operatorname{Aut}(G)$ agree on the vertices of $S$, they agree on all vertices of $G$. That is, $S$ is a determining set if whenever $g$ and $h$ are automorphisms with the property that $g(s)=h(s)$ for all $s \in S$, then $g=h$.

Note that intuitively, a set $S$ is a determining set if and only if every vertex in the graph can be uniquely identified by its own graph properties and its relationship to the vertices in $S$.

Every graph has a determining set. Trivially the set of vertices itself is a determining set. It is also clear that any set containing all but one vertex is a determining set.

Example 1. Figure 1 illustrates the Petersen graph with vertices identified with the 2 -subsets of a 5-set. Using either of the tools of Section 2.2 we can show that the set $S=\{\{1,2\},\{2,4\},\{2,5\}\}$ is a determining set.

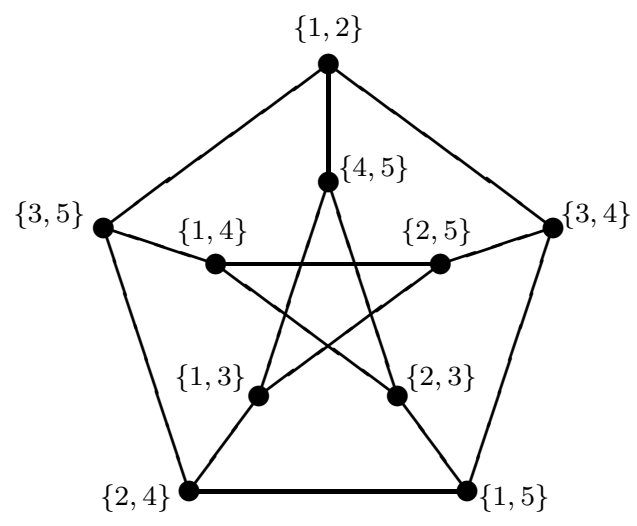

Figure 1: The Petersen Graph 
Definition 2. The determining number of a graph $G$ is the smallest integer $r$ so that $G$ has a determining set of size $r$. Denote this by $\operatorname{Det}(G)$.

We consider a graph with a trivial automorphism group to have determining number 0 . We will see in Example 8 that there are graphs with large automorphism groups but with determining number 1 . At the other end of the spectrum, it is easy to see that since $\operatorname{Det}\left(K_{n}\right)=\operatorname{Det}\left(K_{1, n}\right)=n-1$, determining numbers can be arbitrarily large.

Example 2. Any pair of non-antipodal vertices is a determining set for a cycle. No single vertex has this property. Thus $\operatorname{Det}\left(C_{n}\right)=2$.

We may associate each vertex of the $n$-cube with an $n$-bit binary string so that a pair of vertices is connected by an edge precisely when their associated binary strings differ in exactly one bit.

Example 3. Consider of the set of vertices of the $n$-cube associated with the $n$-bit binary expressions for $0,1,2,4, \ldots, 2^{n-2}$. We can use the tools of Section 2.2 to show that this is a distance determining set and therefore a determining set. Thus $\operatorname{Det}\left(Q_{n}\right) \leq n$ and it is straightforward to show that equality holds when $n=2$ or 3 .

\subsection{Bounds}

Determining sets are useful in investigating graph automorphisms and are particularly useful in proving that all automorphisms of a given graph have been found. If a proposed set of automorphisms takes the determining set to all possible images of itself, then it is indeed the full set of automorphisms. This links the size of a determining set to the size of the automorphism group.

Proposition 1. Let $G$ be a graph with $n$ vertices and determining number $r$. Then $(n-r) ! \leq \frac{n !}{|\operatorname{Aut}(G)|}$.

Proof. Since each automorphic image of the determining set must be unique and there are precisely $\frac{n !}{(n-r) !}$ different ordered $r$-subsets of $V(G)$, there are at most $\frac{n !}{(n-r) !}$ different automorphisms of $G$.

Proposition 2. Let $G$ be a graph with $n$ vertices, maximum orbit size $s$, and determining number $r$. Then $r \geq \log _{s}|\operatorname{Aut}(G)| \geq \log _{n}|\operatorname{Aut}(G)|$.

Proof. Each of the $r$ vertices in the determining set has at most $s$ vertices to which it can be sent by an automorphism. Each automorphism is uniquely determined by where these vertices are sent. Thus there are at most $s^{r}$ automorphisms. Thus $|\operatorname{Aut}(G)| \leq s^{r} \Longrightarrow r \geq$ $\log _{s}|\operatorname{Aut}(G)|$.

Proposition 1 provides the better bound when the graph is transitive, while Proposition 2 gives the better bound when the maximum orbit size is small compared to the total number of vertices. These are illustrated in the following examples. 
Example 4. $K_{10}$ has determining number 9 and this matches the lower bound obtained from Proposition 1. However, Proposition 2 yields a less accurate lower bound of 7 .

Example 5. Let $G$ be $K_{10}$ with a path of length 19 attached to each vertex. The orbit size, automorphism group, and determining number are the same as for $K_{10}$ itself. However the number of vertices in the graph is 200. The lower bound from Proposition 2 is still 7 , which is significantly better here than the lower bound of 3 obtained from Proposition 1.

Example 6. Either of Proposition 1 or Proposition 2 can be used to show that the determining number of the Petersen graph is at least 3. Since we found a determining set of size 3 in Example 1, the determining number of the Petersen graph is 3.

\section{$2.2 \quad$ Finding and Verifying Determining Sets}

We want to be able to build determining sets and to tell when a set is determining. Here we introduce two tools for these purposes.

For any subset $S \subseteq V(G), \operatorname{Stab}(S)=\{g \in \operatorname{Aut}(G) \mid g(v)=v, \forall v \in S\}$. Note that $\operatorname{Stab}(S)=\cap_{v \in S} \operatorname{Stab}(v)$. This is the pointwise stabilizer of $S$, and will be referred to simply as the stabilizer.

Proposition 3. Let $S$ be a subset of the vertices of the graph $G$. Then $S$ is a determining set for $G$ if and only if $\operatorname{Stab}(S)=\{i d\}$.

Proof. If $S$ is a determining set then whenever $g \in \operatorname{Aut}(G)$ fixes each $s \in S, g=i d$. Thus $\operatorname{Stab}(S)=\{i d\}$. Further if $\operatorname{Stab}(S)=\{i d\}$ and $g, h \in \operatorname{Aut}(G)$ so that $g(s)=h(s)$ for all $s \in S$ then $h^{-1} g(s)=s$ for all $s \in S \Longrightarrow h^{-1} g=i d \Longrightarrow g=h$.

Frequently the easiest way to obtain a determining set is to find a set with a trivial stabilizer. However, if we want to find a determining set for the purpose of identifying the automorphism group, we need a method that does not rely on automorphisms or stabilizers. One way to do this is to find a set of vertices $S$ with the property that every vertex in the graph can be uniquely identified by its distances from the vertices of $S$. Here we call such a set a distance determining set. These have been studied previously and are sometimes called resolving sets, reference sets, or locating sets. See for example [1, 7].

Definition 3. Let $G$ be a connected graph. Let $S=\left\{s_{1}, \ldots, s_{r}\right\}$ be an ordered subset of the vertices of $G$. The $S$-distance vector of a vertex $v$ is $\left(d\left(v, s_{1}\right), \ldots, d\left(v, s_{r}\right)\right) \in \mathbb{Z}^{r}$ where each distance is the length of the shortest path between the two vertices. A set $S$ is called a distance determining set for $G$ if the $S$-distance vectors of $G$ are distinct.

Suppose we have a distance determining set $S$, an automorphism $\sigma$, and an arbitrary vertex $v$. Since $v$ is uniquely identified by its distances from the vertices of $S$ and $\sigma$ preserves distance, $\sigma(v)$ is uniquely determined by its distances from $\sigma(S)$. Then $S$ is a determining set for $G$. Thus we have proved the following.

Proposition 4. Let $S$ be a subset of the vertices of the connected graph $G$. If $S$ is a distance determining set for $G$ then it is a determining set for $G$. 
Restricting ourselves to connected graphs does no harm here. If a graph is not connected, we can find a distance determining set for each component. The union of these is a determining set for the graph unless the graph contains multiple isolated vertices. In this case we add all but one of the isolated vertices to the set to make it a determining set.

Example 7. The Petersen graph has determining number 3 and also has distance determining sets of size 3 . However, not all determining sets are distance determining. The determining set given in Example 1 is also a distance determining set, while the set $T=\{\{1,2\},\{2,3\},\{3,4\}\}$ is determining but not distance determining. (The vertices $\{1,3\}$ and $\{2,4\}$ are both at distance 2 from each of the elements of $T$.)

So even without knowledge of the automorphism group, we can find a determining set for a connected graph simply by finding a distance determining set. Further it is easy to verify that a given set is distance determining. However, a minimum size distance determining set may be strictly larger than a minimum size determining set.

Example 8. In a GRR (graphical regular representation) every vertex has a trivial stabilizer [6]. Thus a GRR has determining number 1 . But unless it is $K_{2}$ there must be (at least) two vertices at distance 1 from the determining vertex and thus the distance vectors are not all distinct. Thus the determining number may be strictly smaller than the size of a smallest distance determining set.

\subsection{Using Orbits}

We can learn about determining sets for a graph by looking at the vertex orbits and their induced subgraphs.

Proposition 5. Let $\mathcal{O}_{1}, \ldots, \mathcal{O}_{k}$ be the vertex orbits of $G$. Let $H_{1}, \ldots, H_{k}$ be the associated induced subgraphs. Let $S_{1}, \ldots, S_{k}$ be determining sets for $H_{1}, \ldots, H_{k}$. Then $S=S_{1} \cup \cdots \cup S_{k}$ is a determining set for $G$.

Proof. Since each $\mathcal{O}_{i}$ is invariant under the action of $\operatorname{Aut}(G), \operatorname{Stab}\left(\mathcal{O}_{i}\right) \triangleleft \operatorname{Aut}(G)$ and $\operatorname{Aut}(G) / \operatorname{Stab}\left(\mathcal{O}_{i}\right)$ acts faithfully on $H_{i}$. Thus we may consider $\operatorname{Aut}(G) / \operatorname{Stab}\left(\mathcal{O}_{i}\right)$ as a subgroup of $\operatorname{Aut}\left(H_{i}\right)$. Since $S_{i}$ determines $H_{i}$ under the action of $\operatorname{Aut}\left(H_{i}\right)$, it also determines $H_{i}$ under the action of $\operatorname{Aut}(G) / \operatorname{Stab}\left(\mathcal{O}_{i}\right)$. The fact that $\operatorname{Stab}\left(S_{i}\right)=\{i d\}$ under the action of $\operatorname{Aut}\left(H_{i}\right)$ implies that $\operatorname{Stab}\left(S_{i}\right)=\operatorname{Stab}\left(\mathcal{O}_{i}\right)$ under the action of $\operatorname{Aut}(G)$.

Since the action of $\operatorname{Aut}(G)$ is faithful, the pointwise stabilizer of $G$ is trivial. Then $\operatorname{Stab}(S)=\operatorname{Stab}\left(S_{1} \cup \cdots \cup S_{k}\right)=\operatorname{Stab}\left(S_{1}\right) \cap \cdots \cap \operatorname{Stab}\left(S_{k}\right)=\operatorname{Stab}\left(\mathcal{O}_{1}\right) \cap \cdots \cap \operatorname{Stab}\left(\mathcal{O}_{k}\right)=$ $\operatorname{Stab}(V(G))=\{i d\}$. Thus $S$ is a determining set for $G$.

Proposition 6. Let $T \subseteq V(G)$ be an invariant set of vertices under $\operatorname{Aut}(G)$ such that $\operatorname{Stab}(T)=\{i d\}$. Let $H$ be the subgraph induced by $T$. Then a determining set for $H$ is a determining set for $G$.

Since $\operatorname{Stab}(T)=\{i d\}, T$ is itself a determining set. However, the point of the proposition is that a determining set for $H$ is a smaller determining set contained within $T$. The proof of Proposition 6 echos that of Proposition 5. 


\section{Determining Sets for Kneser Graphs}

\subsection{Basics and Bounds}

The Kneser graph $K_{n: k}$ has vertices associated with the $k$-subsets of the $n$-set $[n]=$ $\{1, \ldots, n\}$ with edges between pairs of vertices whose associated subsets are disjoint. The literature is almost evenly split between those requiring $n>2 k$ and those only requiring $n \geq 2 k$. Here we will define $K_{n: k}$ only for $n>2 k$. (The case $n=2 k$ would yield a set of disconnected edges whose determining number is $\frac{1}{2}\left(\begin{array}{c}2 k \\ k\end{array}\right)$, half the number of vertices.)

Throughout this section vertices will simultaneously be considered as $k$-subsets and as vertices. Note that elements of the symmetric group $S_{n}$ take $k$-subsets to $k$-subsets, and maintain disjointness and nondisjointness; thus they induce graph automorphisms of $K_{n: k}$. The Erdös-Ko-Rado Theorem shows that all automorphisms of Kneser graphs arise in this way [5]. Thus the automorphism group of $K_{n: k}$ is (isomorphic to) $S_{n}$.

Lemma 1. The set $\left\{V_{1}, \ldots, V_{r}\right\}$ is a determining set for $K_{n: k}$ if and only if there exists no pair of distinct elements $a, b \in[n]$ so that for each $i$ either $\{a, b\} \subseteq V_{i}$ or $\{a, b\} \subseteq V_{i}^{c}$.

Proof. It is easy to see that the stabilizer of a vertex ( $k$-subset) $V$ is the direct product of the symmetric group on $V$ and the symmetric group on the set complement, $V^{c}$. Thus $\left\{V_{1}, \ldots, V_{r}\right\}$ is a determining set if and only if $\operatorname{Stab}\left(V_{1}\right) \cap \cdots \cap \operatorname{Stab}\left(V_{r}\right)=\left(S_{V_{1}} \times S_{V_{1}^{c}}\right) \cap$ $\cdots \cap\left(S_{V_{r}} \times S_{V_{r}^{c}}\right)=\{i d\}$, which is true if and only if there exists no pair of distinct elements $a, b \in[n]$ so that for each $i$ either $\{a, b\} \subseteq V_{i}$ or $\{a, b\} \subseteq V_{i}^{c}$.

We can use Lemma 1 to find sharp upper and lower bounds for $\operatorname{Det}\left(K_{n: k}\right)$.

Proposition 7. Det $\left(K_{n: k}\right) \leq n-k$ and this bound is sharp.

Proof. Let $U_{1}=\{1, \ldots, k\}, U_{2}=\{2, \ldots, k+1\}, \ldots, U_{n-k}=\{n-k, \ldots, n-1\}$. The reader may readily check that for every pair of integers $a, b \in[n]$ there is an integer $i$ so that one of $a, b$ is in $U_{i}$ and the other is in $U_{i}^{c}$. By Lemma 1 this proves that $\left\{U_{1}, \ldots, U_{n-k}\right\}$ is a determining set for $K_{n: k}$.

Clearly $\operatorname{Det}\left(K_{n: 1}\right)=n-1$. Further, we saw in Example 6 that $\operatorname{Det}\left(K_{5: 2}\right)=3$ and we will see in Proposition 14 that $\operatorname{Det}\left(K_{6: 2}\right)=4$. Thus the bound is sharp.

The following proofs of Propositions 8 and 9 for the lower bound were much improved by the referee's suggestion to put them in the context of the vector space $\mathbb{F}_{2}^{n}$ (where $\mathbb{F}_{2}$ is the field of order 2). We will first need a little notation. For each $V \subseteq[n]$ let $\vec{V} \in \mathbb{F}_{2}^{n}$ be the binary $n$-tuple with ones in the coordinate positions corresponding to the elements in $V$ and zeros in the coordinate positions corresponding to the elements not in $V$. We call $\vec{V}$ the characteristic vector of the subset $V$. Given $S=\left\{V_{1}, \ldots, V_{r}\right\} \subseteq V\left(K_{n: k}\right)$, let $M$ be the $r \times n$ matrix whose rows are the characteristic vectors $\vec{V}_{1}, \ldots, \vec{V}_{r}$. We call $M$ the characteristic matrix of $S$. The weight of a vector in $\mathbb{F}_{2}^{n}$ is its number of nonzero coordinates. 
Consider a pair of elements $a, b$ in $[n]$. Let $\vec{x}$ be the characteristic vector for $\{a, b\}$ as a subset of $[n]$. If one of $a, b$ is in $V_{i}$ but the other is not, then the $i^{t h}$ coordinate of $M \vec{x}$ is 1. However, since our arithmetic is modulo 2, if both (or neither) of $a$ and $b$ is in $V_{i}$ then the $i^{t h}$ coordinate of $M \vec{x}$ is 0 . The converse of these statements also hold. Thus there is a weight 2 vector $\vec{x} \in \mathbb{F}_{2}^{n}$ so that $M \vec{x}=\overrightarrow{0}$ if and only if there is a pair of elements $a, b \in[n]$ so that for each $i$ either $\{a, b\} \subseteq V_{i}$ or $\{a, b\} \subseteq V_{i}^{c}$. This immediately translates to the following.

Lemma 2. Let $S=\left\{V_{1}, \ldots, V_{r}\right\} \subseteq V\left(K_{n: k}\right)$. Let $M$ be the characteristic matrix of $S$. The following are equivalent.

(a) $S$ is a determining set for $K_{n: k}$.

(b) The kernel of $M$ (as a linear transformation from $\mathbb{F}_{2}^{n}$ to $\mathbb{F}_{2}^{r}$ ) contains no vector of weight 2 .

(c) No two columns of $M$ are equal.

Proposition 8. $\operatorname{Det}\left(K_{n: k}\right) \geq \log _{2}(n+1)$ and this bound is sharp.

Proof. Suppose that $K_{n: k}$ has a determining set $S=\left\{V_{1}, \ldots, V_{r}\right\}$. Let $M$ be the characteristic matrix of $S$. Let $W$ be the kernel of $M$. By Lemma 2, since $S$ is a determining set, $W$ contains no vector of weight 2 .

Let $\vec{e}_{1}, \ldots, \vec{e}_{n}$ be the standard basis vectors of $\mathbb{F}_{2}^{n}$. For the moment, view $\mathbb{F}_{2}^{n}$ as a finite additive group, $W$ as a subgroup, and consider the cosets of the form $W+\vec{e}_{i}$. First we wish to prove that all the $W+\vec{e}_{i}$ are disjoint (and thus distinct). If some pair is not disjoint, there exists $\vec{w}_{i}, \vec{w}_{j} \in W$ so that $\vec{w}_{i}+\vec{e}_{i}=\vec{w}_{j}+\vec{e}_{j}$. This means that $\vec{e}_{i}+\vec{e}_{j}=\vec{w}_{i}+\vec{w}_{j}$, a weight 2 vector in $W$. Since this is not possible, we can conclude that $\left|\cup_{i=1}^{n} W+e_{i}\right|=n|W|$. Thus $\left|\cup_{i=1}^{n} W+e_{i}\right|=n|W| \leq\left|\mathbb{F}_{2}^{n}\right|=2^{n} \Longrightarrow|W| \leq \frac{2^{n}}{n}$.

The Rank-Nullity Theorem tells us that $2^{\operatorname{rank}(M)}|W|=2^{n}$. Since $M$ has $r$ rows, $\operatorname{rank}(M) \leq r \Longrightarrow 2^{r}|W| \geq 2^{n}$. Combining this with $|W| \leq \frac{2^{n}}{n}$ yields $2^{r} \geq n$.

Suppose that $n=2^{r}$. Then $M$ is the $r \times 2^{r}$ characteristic matrix of a determining set. By Lemma 2 no two columns of $M$ are the same. Thus the columns of $M$ are the $2^{r}$ distinct binary $r$-tuples, the characteristic vectors for the subsets of $[r]$. In this context, row $i$ of $M$ records which subsets of $[r]$ the element $i$ is in. Since each element of $[r]$ is in precisely $2^{r-1}$ subsets, the weight of each row is $2^{r-1}$. Thus $k=2^{r-1}$. But we are not considering the case when $k=\frac{n}{2}$ (and even if we did, it wouldn't have determining number $r$ ). Thus $n \neq 2^{r} \Longrightarrow 2^{r} \geq n+1$ and the result follows.

We see in Proposition 9 below that $K_{2^{r}-1: 2^{r-1}-1}$ has a determining set of size $r$. Thus the bound is sharp, as claimed.

Proposition 9. $\operatorname{Det}\left(K_{2^{r}-1: 2^{r-1}-1}\right)=r$.

Proof. Beginning with a construction similar to the above, let $M$ be the $r \times\left(2^{r}-1\right)$ matrix whose columns are the characteristic vectors for the nonempty subsets of $[r]$. Since each 
element of $[r]$ shows up in exactly $2^{r-1}$ subsets of $[r]$, each row of $M$ has weight $2^{r-1}$. Thus each row has $2^{r}-1-2^{r-1}=2^{r-1}-1$ zeros. Switch all zeros to ones and ones to zeros in $M$ to yield $M^{\prime}$. Then $M^{\prime}$ can be considered a characteristic matrix in which each row has weight $2^{r-1}-1$. The kernel of $M^{\prime}$ has a vector of weight 2 if and only if two columns of $M^{\prime}$ are equal. But by construction all columns of $M$, and therefore of $M^{\prime}$, are distinct. Thus $M^{\prime}$ is a characteristic matrix for a determining set for $K_{2^{r}-1: 2^{r-1}-1}$ whose characteristic vectors are the rows of $M^{\prime}$. Thus $K_{2^{r}-1: 2^{r-1}-1}$ has a determining set of size $r$. Combining this result with that of Proposition 8 yields the result.

\subsection{Kneser Graphs with Given Determining Number}

Lemma 1 can help us find all Kneser graphs with determining number 2,3 or 4 . For any $V \subset[n]$ let $V^{1}=V$ and $V^{-1}=V^{c}$. With this notation, the statement 'There exists no pair of distinct elements $a, b \in[n]$ so that for each $i$ either $\{a, b\} \subseteq V_{i}$ or $\{a, b\} \subseteq V_{i}^{c}$ '. is equivalent to the statement 'There is no assignment of \pm 1 to the $e_{i}$ so that $\left|V_{1}^{e_{1}} \cap \cdots \cap V_{r}^{e_{r}}\right| \geq 2$.' Thus Lemma 1 is equivalent to the following.

Lemma $\mathbf{1}^{\prime}$. The set $\left\{V_{1}, \ldots, V_{r}\right\}$ is a determining set for $K_{n: k}$ if and only if for all possible assignments of \pm 1 to $e_{1}, \ldots, e_{r},\left|V_{1}^{e_{1}} \cap \cdots \cap V_{r}^{e_{r}}\right| \leq 1$.

Using Lemma $1^{\prime}$ requires that we be able to compute $\left|V_{1}^{e_{1}} \cap \cdots \cap V_{r}^{e_{r}}\right|$. To accomplish this we use a natural generalization of the fact that $\left|A \cap B^{c}\right|=|A|-|A \cap B|$.

Let $V_{1}, \ldots, V_{r}$ be vertices of $K_{n: k}$. For each nonempty $U \subseteq[r]$, let $A_{U}=\cap_{i \in U} V_{i}$. Further let $A_{\emptyset}=[n]$. Then $\left|A_{\emptyset}\right|=n$, and for each $i,\left|A_{\{i\}}\right|=\left|V_{i}\right|=k$. For each $T \subseteq[r]$, let $E_{T}=\left|V_{1}^{e_{1}} \cap \cdots \cap V_{r}^{e_{r}}\right|$ where $e_{i}=1$ when $i \in T$ and $e_{i}=-1$ otherwise. Proposition 10 below gives a formula for $E_{T}$ in terms of the $\left|A_{U}\right|$. For example, if $r=3$, we can easily see that

$$
\begin{aligned}
E_{\{1\}} & =\left|V_{1} \cap V_{2}^{c} \cap V_{3}^{c}\right| \\
& =\left|V_{1}\right|-\left|V_{1} \cap V_{2}\right|-\left|V_{1} \cap V_{3}\right|+\left|V_{1} \cap V_{2} \cap V_{3}\right| \\
& =\left|A_{\{1\}}\right|-\left|A_{\{1,2\}}\right|-\left|A_{\{1,3\}}\right|+\left|A_{\{1,2,3\}}\right| .
\end{aligned}
$$

On the other hand, Proposition 11 gives a formula for $\left|A_{U}\right|$ in terms of the $E_{T}$. For example, starting with the formula from Proposition 10 it is easy to see that when $r=3$,

$$
\begin{aligned}
\left|A_{\{1,2,3\}}\right| & =E_{\{1,2,3\}}, \\
\left|A_{\{1,2\}}\right| & =E_{\{1,2\}}+E_{\{1,2,3\}}, \\
\left|A_{\{1\}}\right| & =E_{\{1\}}+E_{\{1,2\}}+E_{\{1,3\}}+E_{\{1,2,3\}} .
\end{aligned}
$$

The formula from Proposition 10 is useful in checking whether a set is a determining set. However, the formula from Proposition 11 enables us to start with an assignment of allowable values (each 0 or 1 ) for the $E_{T}$ and see which $\left|A_{U}\right|$ arise. This formula can be used with the fact that all $A_{\{i\}}$ have the same cardinality to rule out certain combinations of values for the $E_{T}$. For those that we don't rule out, we can then use the formula to find $n$ and $k$ for a potential Kneser graph with the desired size determining set (Propositions 12,13 , and 14). These results are worked out carefully after the statements and proofs of Propositions 10 and 11. 
However, we could also use Proposition 11 to compute or bound certain determining numbers and to verify certain determining sets. In particular, we could use this formula to find a maximum $n$ for a given size determining set and use this to find a sharp lower bound for the determining number of a Kneser graph (providing an alternate proof of Proposition 8). We could also use Proposition 11 to find a closed expression for the $\left|A_{U}\right|$ given a particularly nice set of values for the $E_{T}$, and use this to verify that a given set is a determining set (providing an alternate proof Proposition 9).

Proposition 10. For any $T \subseteq[r]$,

$$
E_{T}=\left|A_{T}\right|+\cdots+(-1)^{j} \sum_{U_{j}}\left|A_{U_{j}}\right|+\cdots+(-1)^{r-|T|}\left|A_{[r]}\right|,
$$

where the $U_{j}$ range over all subsets of $[r]$ that contain $T$ and have cardinality $|T|+j$.

Proof. Proof by induction on $r$. Let $T \subseteq[r]$.

Case 1: Suppose that $r \in T$. Then $E_{T}=\left|V_{1}^{e_{1}} \cap \cdots \cap V_{r-1}^{e_{r-1}} \cap V_{r}\right|$. Let $D=V_{1}^{e_{1}} \cap \cdots \cap V_{r-1}^{e_{r-1}}$. Then $E_{T}=\left|D \cap V_{r}\right|$.

Let $T^{\prime}=T-\{r\}$. Then $T^{\prime}$ is a subset of $[r-1]$ and by the inductive hypothesis $|D|=\left|A_{T^{\prime}}\right|+\cdots+(-1)^{j} \sum_{U_{j}}\left|A_{U_{j}}\right|+\cdots+(-1)^{r-1-\mid T^{\prime}}\left|A_{[r-1]}\right|$ where the $U_{j}$ range over all subsets of $[r-1]$ that contain $T^{\prime}$ and have cardinality $\left|T^{\prime}\right|+j$.

Let $\ell=\left|A_{T^{\prime}} \cap V_{r}\right|+\cdots+(-1)^{j} \sum_{U_{j}}\left|A_{U_{j}} \cap V_{r}\right|+\cdots+(-1)^{r-1-\left|T^{\prime}\right|}\left|A_{[r-1]} \cap V_{r}\right|$. By studying the possible contribution of an arbitrary element $x \in[n]$ we will see that $\ell=\left|D \cap V_{r}\right|=E_{T}$.

If $x \in D$ and $x \in V_{r}$ then $x \in A_{U_{i}} \cap V_{r} \Longleftrightarrow x \in A_{U_{i}}$. Thus $x$ contributes the same to $\ell$ as it does to $|D|=\left|A_{T^{\prime}}\right|+\cdots+(-1)^{j} \sum_{U_{j}}\left|A_{U_{j}}\right|+\cdots+(-1)^{r-1-\left|T^{\prime}\right|}\left|A_{[r-1]}\right|$, which is 1 .

If $x \in D$ but $x \notin V_{r}$ then $x$ contributes nothing to any $\left|A_{U_{i}} \cap V_{r}\right|$ and thus does not contribute to $\ell$.

If $x \notin D$ and $x \in V_{r}$ then $x \in A_{U_{i}} \cap V_{r} \Longleftrightarrow x \in A_{U_{i}}$. Thus $x$ contributes the same to $\ell$ as it does to $|D|$, which is 0 .

If $x \notin D$ and $x \notin V_{r}$ then $x$ contributes nothing to any $\left|A_{U_{i}} \cap V_{r}\right|$ and thus does not not contribute to $\ell$.

Thus $\ell$ counts precisely those elements in $D \cap V_{r}$ and this count is $E_{T}$. That is,

$$
E_{T}=\left|A_{T^{\prime}} \cap V_{r}\right|+\cdots+(-1)^{j} \sum_{U_{j}}\left|A_{U_{j}} \cap V_{r}\right|+\cdots+(-1)^{r-1-\left|T^{\prime}\right|}\left|A_{[r-1]} \cap V_{r}\right|
$$

where the $U_{j}$ range over all subsets of $[r-1]$ that contain $T^{\prime}$ and have cardinality $\left|T^{\prime}\right|+j$. Note that $A_{T^{\prime}} \cap V_{r}=A_{T^{\prime} \cup\{r\}}=A_{T}$ and for each $U_{j}$ containing $T^{\prime}$ of cardinality $\left|T^{\prime}\right|+j$, $A_{U_{j}} \cap V_{r}=A_{U_{j} \cup\{r\}}$ where $U_{j} \cup\{r\}$ contains $T$ and has cardinality $|T|+j$. Thus we can rewrite our sum as

$$
E_{T}=\left|A_{T}\right|+\cdots+(-1)^{j} \sum_{U_{j}}\left|A_{U_{j}}\right|+\cdots+(-1)^{r-|T|}\left|A_{[r]}\right|
$$


where the $U_{j}$ range over all subsets of $[r]$ that contain $T$ and have cardinality $|T|+j$. Thus we have our result when $r \in T$.

Case 2: Suppose that $r \notin T$. Then $E_{T}=\left|V_{1}^{e_{1}} \cap \cdots \cap V_{r-1}^{e_{r-1}} \cap V_{r}^{c}\right|$. Let $T^{\prime}=T-\{r\}=T$ and, as in Case 1 , let $D=V_{1}^{e_{1}} \cap \cdots \cap V_{r-1}^{e_{r}-1}$. Recall that by the inductive hypothesis $|D|=\left|A_{T^{\prime}}\right|+\cdots+(-1)^{j} \sum_{U_{j}}\left|A_{U_{j}}\right|+\cdots+(-1)^{r-1-\left|T^{\prime}\right|}\left|A_{[r-1]}\right|$ where the $U_{j}$ range over subsets of $[r-1]$ that contain $T^{\prime}$ and have cardinality $\left|T^{\prime}\right|+j$. Since $T^{\prime}=T$ we can rewrite this as

$$
|D|=\left|A_{T}\right|+\cdots+(-1)^{j} \sum_{U_{j}}\left|A_{U_{j}}\right|+\cdots+(-1)^{r-1-|T|}\left|A_{[r-1]}\right| .
$$

Again as in Case 1,

$$
\left|D \cap V_{r}\right|=\left|A_{T^{\prime}} \cap V_{r}\right|+\cdots+(-1)^{j} \sum_{U_{j}}\left|A_{U_{j}} \cap V_{r}\right|+\cdots+(-1)^{r-1-\left|T^{\prime}\right|}\left|A_{[r-1]} \cap V_{r}\right| .
$$

But this can also be rewritten as

$$
\left|D \cap V_{r}\right|=\left|A_{T \cup\{r\}}\right|+\cdots+(-1)^{j} \sum_{U_{j}}\left|A_{U_{j} \cup\{r\}}\right|+\cdots+(-1)^{r-1-|T|}\left|A_{[r]}\right| .
$$

Bringing these together yields

$$
\begin{aligned}
E_{T}= & \left|D \cap V_{r}^{c}\right| \\
= & |D|-\left|D \cap V_{r}\right| \\
= & \left(\left|A_{T}\right|+\cdots+(-1)^{j} \sum_{U_{j}}\left|A_{U_{j}}\right|+\cdots+(-1)^{r-1-|T|}\left|A_{[r-1]}\right|\right) \\
& -\left(\left|A_{T \cup\{r\}}\right|+\cdots+(-1)^{j} \sum_{U_{j}}\left|A_{U_{j} \cup\{r\}}\right|+\cdots+(-1)^{r-1-|T|}\left|A_{[r]}\right|\right) \\
= & \left(\left|A_{T}\right|+\cdots+(-1)^{j} \sum_{U_{j}}\left|A_{U_{j}}\right|+\cdots+(-1)^{r-1-|T|}\left|A_{[r-1]}\right|\right) \\
& +\left(-\left|A_{T \cup\{r\}}\right|+\cdots+(-1)^{j+1} \sum_{U_{j}}\left|A_{U_{j} \cup\{r\}}\right|+\cdots+(-1)^{r-|T|}\left|A_{[r]}\right|\right),
\end{aligned}
$$

where the $U_{j}$ range over subsets of $[r-1]$ that contain $T$ and have cardinality $|T|+j$.

Thus we can consider the first half of this sum to be taken over all subsets of $[r]$ that contain $T$ but not $r$, and the second half to be taken over all subsets of $[r]$ that contain both $T$ and $r$. In both cases the sign is positive if the subset length minus $|T|$ is even, and negative otherwise. Thus this is an alternating sum over all subsets of $[r]$ containing $T$ with appropriate signs. Thus we have our result when $r \notin T$.

Proposition 11. $\left|A_{U}\right|=\sum_{T} E_{T}$ where the $T$ range over all subsets of $[r]$ containing $U$.

Proof. For an arbitrary $r$, the proof is by induction on $r-|U|$. It is clearly true when $U=\emptyset$.

Let $U$ be fixed. By our previous proposition

$$
E_{U}=\left|A_{U}\right|+\cdots+(-1)^{j} \sum_{U_{j}}\left|A_{U_{j}}\right|+\cdots+(-1)^{r-|U|}\left|A_{[r]}\right|
$$


where the $U_{j}$ range over all subsets of $[r]$ with cardinality $|U|+j$. Thus

$$
\left|A_{U}\right|=E_{U}+\cdots+(-1)^{j+1} \sum_{U_{j}}\left|A_{U_{j}}\right|+\cdots+(-1)^{r-|U|+1}\left|A_{[r]}\right| .
$$

For each $j>0, r-\left|U_{j}\right|<r-|U|$. Thus by the inductive hypothesis each $\left|A_{U_{j}}\right|=\sum_{T} E_{T}$ where the $T$ range over all subsets of $[r]$ containing $U_{j}$.

Fix an arbitrary $U_{j} \subseteq[r]$ so that $U \subseteq U_{j}$. Our question is: What is the net number of copies of $E_{U_{j}}$ that show up on the right hand side of Equation (1)?

This $U_{j}$ shows up once in every $\left|A_{U_{i}}\right|=\sum_{T} E_{T}$ where $U \subseteq U_{i} \subseteq U_{j} . E_{U_{j}}$ is being added if $i=\left|U_{i}\right|-|U|$ is odd, and it is being subtracted if $i$ is even. For each $i=1, \ldots, j$ there are $\left(\begin{array}{l}j \\ i\end{array}\right)$ sets of cardinality $|U|+i$ fitting these conditions. Thus the net number of copies of $E_{U_{j}}$ on the right hand side is $\left(\begin{array}{l}j \\ 1\end{array}\right)-\left(\begin{array}{l}j \\ 2\end{array}\right)+\cdots+(-1)^{j+1}\left(\begin{array}{l}j \\ j\end{array}\right)$. But this is 1 plus an alternating sum of the $j^{\text {th }}$ row of Pascal's triangle. Thus, for every $U_{j}$ containing $U$, there is a net of one copy of $E_{U_{j}}$ on the right hand side of Equation (1).

Proposition 12. The only Kneser graph with determining number 2 is $K_{3: 1}$.

The proof for the above is an even simpler version of the proof of the following.

Proposition 13. The only Kneser graphs with determining number 3 are $K_{4: 1}, K_{5: 2}$ and $K_{7: 3}$.

Proof. If $K_{n: k}$ has a determining set of size 3 , then there exist vertices $V_{1}, V_{2}, V_{3}$ yielding $E_{T} \in\{0,1\}$ for each $T \subseteq\{1,2,3\}$. Using Proposition 11 we can check all $2^{2^{3}}$ possible assignments of values to the $E_{T}$ to determine those for which $A_{\{1\}}=A_{\{2\}}=A_{\{3\}}$. For each such assignment we can compute the associated $n$ and $k$ values. Some of these we reject because $k \geq \frac{n}{2}$ or because $k=0$; some of these we reject because they are known to have determining number 1 or 2 . Those that are left are then candidates for Kneser graphs with determining number 3 .

In this case, the only possibilities that resulted were $K_{4: 1}, K_{5: 2}$ and $K_{7: 3}$.

There is a determining set of size 3 for $K_{4: 1}$ that consists of all but one of the vertices. There is a determining set of size 3 for $K_{5: 2}$ given in Example 1, and one for $K_{7: 3}$ can be constructed as in Proposition 9. Thus each of these does indeed have a determining set of size 3 and therefore determining number 3 .

Proposition 14. The only Kneser graphs with determining number 4 are $K_{5: 1}, K_{6: 2}$, $K_{7: 2}, K_{8: 3}, K_{9: 3}, K_{9: 4}, K_{10: 4}, K_{11: 4}, K_{11: 5}, K_{12: 5}, K_{13: 6}, K_{15: 7}$.

Proof. As in the proof above, we can try all $2^{2^{4}}$ values for $E_{\emptyset}, \ldots, E_{[4]}$ to find a complete list of candidates for $K_{n: k}$ with determining number 4 . The only viable candidates are those listed in the proposition. The following are determining sets of size 4 for each of them. The interested reader can verify that these are determining sets by computing the $E_{T}$. The exceptionally careful reader can write a computer program to verify that these are indeed all the possibilities. 
- There is a determining set of size 4 for $K_{5: 1}$ that consists of all but one of the vertices.

- $\{\{1,2\},\{1,3\},\{4,5\},\{4,6\}\}$ is a determining set for each of $K_{6: 2}$ and $K_{7: 2}$.

- $\{\{1,2,3\},\{1,4,5\},\{2,6,7\},\{4,6,8\}\}$ is a determining set for $K_{8: 3}$ and $K_{9: 3}$.

- $\{\{1,2,3,4\},\{1,5,6,7\},\{1,2,5,8\},\{3,6,8,9\}\}$ is a determining set for $K_{9: 4}$ and $K_{10: 4}$.

- $\{\{1,2,3,4\},\{1,5,6,7\},\{2,5,8,9\},\{3,6,8,10\}\}$ is a determining set for $K_{11: 4}$.

- $\{\{1,2,3,4,5\},\{1,2,6,7,8\},\{1,3,6,9,10\},\{2,4,7,9,11\}\}$ is a determining set for $K_{11: 5}$ and $K_{12: 5}$.

- $\{\{1,2,3,4,5,6\},\{1,2,3,7,8,9\},\{1,4,5,7,10,11\},\{2,4,6,8,10,12\}\}$ is a determining set for $K_{13: 6}$.

- A determining set of size 4 for $K_{15: 7}$ can be constructed as in Proposition 9 .

\section{Open Questions}

Question 1. Can the difference between the determining number of a graph and the size of a smallest distance determining set for a graph be arbitrarily large?

Question 2. We know that $\operatorname{Det}\left(K_{n: k}\right)=n-k$ for $K_{n: 1}$ for any $n$, and for $K_{5: 2}$, and $K_{6: 2}$. However, $\operatorname{Det}\left(K_{7: 2}\right)=4 \neq 7-2$. Is there an infinite family of Kneser graphs with $k \geq 2$ for which $\operatorname{Det}\left(K_{n: k}\right)=n-k$ ?

Acknowledgment: I would like to thank the referee for the suggestions that added elegance to the proofs of Propositions 8 and 9.

\section{References}

[1] Gary Chartrand, Linda Eroh, Mark A. Johnson, and Ortrud R. Oellermann. Resolvability in graphs and the metric dimension of a graph. Discrete Appl. Math., 105(1-3):99-113, 2000.

[2] Karen L. Collins and Joshua D. Laison. Personal communication.

[3] David Erwin and Frank Harary. Destroying automorphisms by fixing nodes. Manuscript, August 2004.

[4] Courtney R Gibbons and Joshua D. Laison. Personal communication.

[5] Chris Godsil and Gordon Royle. Algebraic Graph Theory, volume 207 of Graduate Texts in Mathematics. Springer-Verlag, New York, 2001.

[6] R. L. Graham, M. Grötschel, and L. Lovász, editors. Handbook of Combinatorics. Vol. 1, 2. Elsevier Science B.V., Amsterdam, 1995.

[7] Peter J. Slater. Leaves of trees. Congressus Numerantium, 14:549-559, 1975. 